\title{
Research of the ECAL calorimeter used in the COMET experiment
}

\author{
V.Kalinnikov, E.Velicheva \\ Joint Institute for Nuclear Research, 141980 Dubna, Russia
}

Received November 28, 2014

\begin{abstract}
Based on the real optical model received with the Geant4 simulation, the optimal structure and geometry of the COMET calorimeter was developed, the influence of the shower was studied and energy deposition and calorimeter energy resolution (equal to $4.8 \%$ for the LYSO crystal calorimeter and $6.0 \%$ for the GSO crystal calorimeter) was defined. There was suggested and experimentally tested the algorithm of spatial reconstruction of events in the calorimeter allowing to improve the determination accuracy of the energy deposition value in 2.8 times.

Keywords: ECAL calorimeter, Algorithm of spatial reconstruction of events, Geant4 simulation.

На основе полученной реальной оптической модели, на базе Geant4 моделирования разработана оптимальная структура и геометрия СОМЕТ калориметра, изучено влияние ливня, определено энерговыделение и энергетическое разрешение калориметра, равное $4,8 \%$ и $6,0 \%$ для калориметра на LYSO на GSO кристаллах, соответственно. Предложен и экспериментально проверен алгоритм пространственной реконструкции событий в калориметре, позволяющий улучшить точность определения значения величины энерговыделения в калориметре примерно в 2,8 раз.
\end{abstract}

\footnotetext{
Дослідження ECAL калориметра для експерименту COMET. В.Калинников, Е.Велічева.

На основі отриманої реальної оптичної моделі, на базі Geant4 моделювання розроблено оптимальну структуру та геометрію COMET калориметра, вивчено вплив зливи, визначено енерговиділення та енергетичний розподіл калориметра, який дорівнює $4.8 \%$ i 6.0 \% для калориметра на LYSO на GSO кристалах, відповідно. Запропоновано й експериментально перевірено алгоритм просторової реконструкції подій у калориметрі, що дозволяє поліпшити точність визначення значення величини енерговиділення у калориметрі приблизно в 2,8 разів.
}

\section{Introduction}

The discovery of neutrino oscillations has given a new impetus to the experiments on the search for rare processes, in which there is a violation of the law of conservation of lepton number in the sector of charged leptons (CLFV). The main processes which are accessible for CLFV are the decays, $\mu \rightarrow e \gamma, \mu \rightarrow e e e, \mu^{-} \rightarrow e^{-}$conversion, neutrinoless double decay $\beta$ and others.

When a negative muon is stopped by some material, it is trapped by an atom, and a muonic atom is formed. After it cascades down energy levels in the muonic atom, the muon is bound in its $1 s$ ground state. There are two modes of decay: decay in orbit $\mu^{-} \rightarrow e^{-} v_{\mu} \bar{v}_{\mathrm{e}}$ or captured by a nucleus of mass number $A$ and atomic number $Z$, namely, $\mu^{-}+N(A, Z) \rightarrow v_{\mu}+$ $+N(A, Z-1)$. However, in the context of physics beyond the Standard Model, the exotic process of neutrinoless muon capture, such as $\mu^{-}+N(A, Z) \rightarrow e^{-}+N(A, Z)$ is also expected. This process is called $\mu^{-} \rightarrow e^{-}$conversion in a muonic atom [1]. The sensitivity of the 
$\mu^{-} \rightarrow e^{-}$conversion process is defined as the ratio

$$
R_{\mu e}=\frac{\Gamma\left(\mu^{-}+N(A, Z) \rightarrow e^{-}+N(A, Z)\right)}{\Gamma\left(\mu^{-}+N(A, Z) \rightarrow v_{\mu}+N(A, Z-1)\right)} .
$$

The process of neutrinoless $\mu^{-} \rightarrow e^{-}$conversion is one of the most sensitive to search the violation of the lepton number of processes. The latest search for $\mu^{-} \rightarrow e^{-}$conversion was performed by the SINDRUM-II collaboration at PSI and is equal to $R_{\mu e} \leq 7 \cdot 10^{-13}$ [2]. COMET aims to search this process for an atom of aluminum $\mu^{-}+$ $\mathrm{Al} \rightarrow e^{-}+\mathrm{Al}$ at a sensitivity below $10^{-17}$ [3].

COMET will use a bunched proton beam, slow-extracted from the J-PARC (Tokyo). Beam bunching will be necessary to reject beam-related backgrounds. The COMET experimental setup consists of high magneticfield solenoids for pion capture, C-shaped curved solenoids with momentum selection, and a C-shaped curved solenoid spectrometer to remove charged-particle backgrounds of low momentum, so as, to reduce single counting rates of the detectors, and to maximize the transmission of $\mu^{-} \rightarrow e^{-}$conversion signals. The COMET detector section consists of an electron tracker for the definition of the electron momentum and an electromagnetic calorimeter (ECAL) which is made on crystals. The calorimeter is located behind the tracker. Electrons which pass through the tracker are absorbed in the calorimeter and if the energy deposited lies within a small window around $105 \mathrm{MeV}$, a trigger signal is generated.

The ECAL calorimeter is one of the most important parts of the COMET setup, from which depends the possibility of implementing the tasks of the whole experiment. Therefore, there are high requirements for parameters of the calorimeter. The calorimeter should have an energy resolution of better than $5 \%$, as events that should be recorded in this experiment lie in a narrow energy region (within $105 \pm 0.5 \mathrm{MeV}$ ), and an energy-correlated position coordinate accuracy of about $1 \mathrm{~cm}$ (rms). ECAL should solve the following tasks:

- the measurement of the electrons energy very exactly;

- the production time trigger signal for recording system events;

- to do the correlation position of the track of an electron and its energy;

- to identify the electrons, neutrons and low energy gammas.

\section{Aims and tasks for the research of the ECAL calorimeter}

The main aim of the research was to determine the expected parameters of the ECAL calorimeter using different types of crystals (the COMET experiment considers two candidates - GSO and LYSO crystals) and reflective materials and the determination of optimal structure and geometry of the calorimeter. All studies were made using the Geant4 simulation [4] and experimental measurements of crystals on a measuring setup [5] and beam test of the calorimeter prototype at the electron beam of an accelerator in Tohoku (Japan, March 2014) [6].

To closely match the simulation results and real experimental measurements it is necessary that the optical model, which is included in the simulation, is described the properties of GSO and LYSO crystals a well. To realize this aim the following tasks were solved:

- the experimental study of optical parameters of long GSO and LYSO crystals on a specially created a precision measurement setup;

- the SLitrani simulation of GSO and LYSO crystals for more precise definition and obtaining optical parameters required for the Geant4 simulation of the ECAL calorimeter;

- the comparison of simulation results and experimental data to evaluate the accuracy of the optical model.

When the research of the ECAL calorimeter using Geant4 following tasks were solving:

- the evaluation of the energy resolution and energy deposition in the calorimeter with the received real-optical parameters of LYSO and GSO crystals;

- the simulation of structure and geometry of the calorimeter.

To obtain more precise values of real parameters of the COMET ECAL calorimeter the experimental measurements of the calorimeter prototypes (the matrix of $7 \times 7$ crystals prepared on GSO and LYSO crystals) at the electron beam of the accelerator in Tohoku were made.

\section{Experimental study of optical parameters of long GSO and LYSO crystals}

The crystals used in the calorimeter should have high-energy resolution, large 
Table 1. Mean values and standard deviation of the measured optical parameters for 50 LYSO crystals

\begin{tabular}{|c|c|c||}
\hline \multicolumn{2}{|c|}{ Parameter } & Mean value \\
\hline 1 & $\begin{array}{c}\text { Coefficient nonuniformity, \% } \\
\mathrm{cm}^{-1}\end{array}$ & $1.44 \pm 0.03$ \\
2 & $\begin{array}{c}\text { Losses coefficient of light } \\
\text { output, \% cm }\end{array}$ & $1.82 \pm 0.05$ \\
3 & Energy resolution, \% & $8.85 \pm 0.06$ \\
4 & Relative light output, $\mathrm{mV}$ & $158.5 \pm 2.5$ \\
\hline \hline
\end{tabular}

light output for long (of $12-15 \mathrm{~cm}$ ) crystals, fast decay time for reliable separation pile-up events and high radiation resistance. There are two candidates with the desired values of optical parameters - LYSO and GSO crystals $[7,8]$. So, the experimental study and measurement of optical parameters of LYSO and GSO crystals for the comparative evaluation of candidates and to obtain more precise optical properties were made. The results are published in [5]. Also the checking and calibration of 50 LYSO $\left(20 \times 20 \times 120 \mathrm{~mm}^{3}\right)$ crystals for calorimeter prototype for beam test measurement at electron beam of an accelerator in Tohoku was made [9].

In Table 1 the mean values and standard deviation of the measured optical parameters for 50 LYSO crystals from SAINT-GOBAIN (France) are given. In Table 2 the comparative results of experimental measurements of optical parameters of GSO crystals performed at JINR (Dubna) and KEK (Tsukuba, Japan) are given [10].

\section{The SLitrani simulation of GSO and LYSO crystals}

The researches of LYSO and GSO crystals have been made by using package SLitrani (Super Light Ray Tracing in ANIsotropic media) to obtain more precise optical model of the calorimeter [11]. The Slitrani package usually uses for Monte Carlo simulating of light propagation in isotropic and anisotropic media environments. In this program, the optical parameters of medium define as functions of wavelength, photon energy, and other values and allow describing the processes inside the investigated media more accurately.

The main tasks of the simulation using the package SLitrani are:

- the obtaining or clarification of optical parameters of LYSO and GSO crystals
Table 2. Comparative results of experimental measurements of GSO crystals optical parameters

\begin{tabular}{|c|c|c|c|}
\hline \multicolumn{2}{|r|}{ Parameter } & JINR & KEK \\
\hline 1 & $\begin{array}{c}\text { Energy resolution at } \\
1274.5 \mathrm{keV}, \%\end{array}$ & 9.5 & 9.8 \\
\hline 2 & $\begin{array}{l}\text { Decay time fast } \\
\text { component, ns }\end{array}$ & $60 \pm 0.5$ & 53 \\
\hline 3 & $\begin{array}{l}\text { Decay time slow } \\
\text { component, ns }\end{array}$ & $420 \pm 0.5$ & 400 \\
\hline 4 & Rise time, $\mathrm{ns}$ & $17 \pm 0.03$ & 21 \\
\hline 5 & $\begin{array}{l}\text { Losses of light output } \\
\text { along the crystal length, \% }\end{array}$ & $17.9 \pm 1.2$ & 22 \\
\hline
\end{tabular}

Table 3. Values of the measured and simulated optical parameters of GSO crystals

\begin{tabular}{||c|c|c||}
\hline Optical parameter & $\begin{array}{c}\text { Measured } \\
\text { value }\end{array}$ & $\begin{array}{c}\text { Simulated } \\
\text { value }\end{array}$ \\
\hline Decay time, ns & $420 \pm 0.5$ & 430 \\
Rise time, ns & $17 \pm 0.03$ & 15 \\
\hline
\end{tabular}

and reflective materials necessary for the Geant4 simulation of the calorimeter;

- the research of the real losses of energy (photons) along the crystal length;

- the research of photons losses in reflective materials of the wrapper (Teflon, Mylar, etc).

The energy spectrum and spectrum of scintillation of the crystal as functions of photon energy (wavelength); decay time, photons losses along the crystal length, photon losses in reflective materials of the wrapper, total photons losses of and quantum efficiency detection as a functions of wavelength were obtained in the result of the simulation. These parameters were used in the Geant4 simulation of the ECAL calorimeter.

The values of main optical parameters of GSO crystal obtained using the SLitrani simulation and experimental measurements are given in Fig. 1 and Table 3. The analysis of the results (Table 3) shows that the difference between the simulated and measured values of light output losses along the crystal length is about $3 \%$. In addition, there is a good agreement between the simulated and measured values of decay and rise time. Thus, the adequacy of the parameters of the optical model and real parameters of GSO crystal are confirmed. 

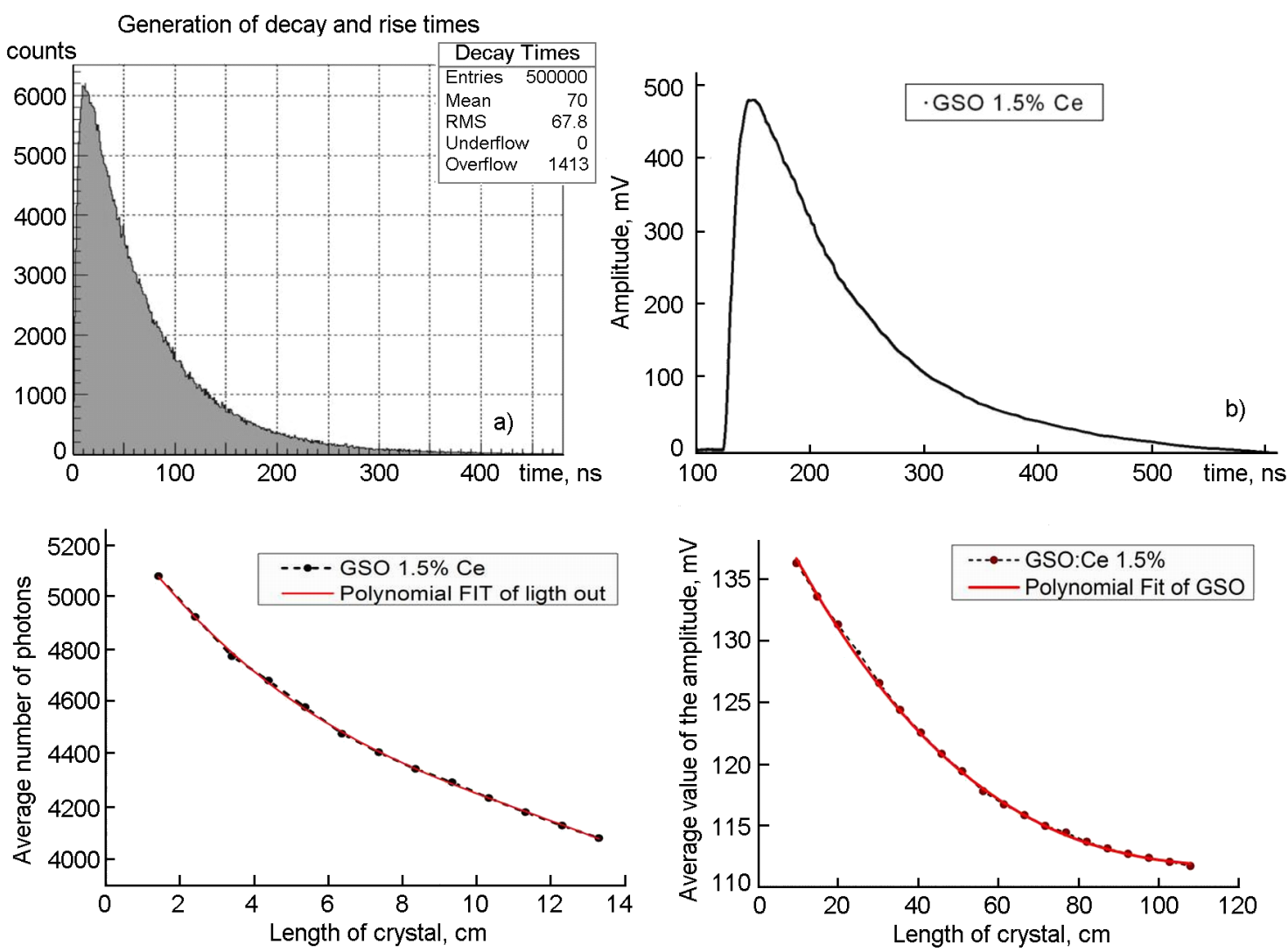

Fig. 1. Values of main optical parameters of GSO crystal obtained using the SLitrani simulation and experimental measurements: a) the time spectrum of the simulated signal; b) the time spectrum of the measured signal; c) the simulated photons losses along the crystal length; d) the measured photons losses along the crystal length.

\section{Geant4 simulation}

The study of the ECAL calorimeter parameters for different types of the crystals and reflective materials was made based on the Geant4 simulation. The main parameters of crystals and physical processes included in the Geant4 simulation are given in Table 4. In addition, in Geant4 simulations were used the optical parameters of real crystals obtained from the experimental measurements, namely, the slow and fast components of decay time (FastTimeConstant and SlowTimeConstant), the light output (LightOutput). Also, the results of the SLitrani simulation, for example, the spectrum of scintillation (ScintillationYield), length of absorption (AbsLength), refractive index (Rindex) and others were used.

During the simulation of the calorimeter was located in $1 \mathrm{~T}$ uniform magnetic field (as in the COMET experiment). The beam energy spread was $105 \pm 0.5 \mathrm{MeV}$. The beam spot was $1 \mathrm{~cm}^{2} \pm 1 \mathrm{~cm}$.

The simulation of the calorimeter was made for cases when:
- all crystals were without reflective wrapper;

- each crystal was wrapped with Teflon;

- each crystal was wrapped with Teflon, and they are grouped in modules of $2 \times 2$ or $3 \times 3$ crystals, wrapped with Mylar.

The best energy resolution and energy deposition were obtained for the calorimeter on the crystals without reflective wrapper, as in this case took into account the whole electromagnetic shower without the photons losses in the material of Teflon. However, this case did not satisfy the requirements of the calorimeter on the coordinate resolution.

The result of simulation of the calorimeter on crystals wrapped with Teflon presented on Fig. 2c. In this case, there are photons losses in the reflective wrapper. These losses include multiple reflections and scattering between the crystal and material of wrapper, the scattering and absorption of photons in the material of Teflon.

Combining crystals in modules of $2 \times 2$ or $3 \times 3$ crystals, wrapped with Mylar, signifi- 
Table 4. Parameters of crystals and physical processes included in the Geant4 simulation

\begin{tabular}{|c|c|c|c|c|}
\hline \multirow[t]{2}{*}{ Parameter } & \multicolumn{2}{|c|}{ LYSO $(C: 1.5 \%)$} & \multicolumn{2}{|c|}{ GSO $(C: 1.5 \%)$} \\
\hline & Referenced data & Measured & Referenced data & Measured \\
\hline Geometrical sizes & $20 \times 20 \times 120 \mathrm{~mm}^{3}$ & & & \\
\hline Density $\left[\mathrm{g} / \mathrm{cm}^{3}\right]$ & 6.71 & - & 7.4 & - \\
\hline Decay time: Fast/slow component [ns] & $40-60 / 300-600$ & $60 / 470$ & $40-60 / 400-1000$ & $47 / 200$ \\
\hline Number $[Z$ eff $]$ & 59 & - & 66 & - \\
\hline Refraction index & 1.85 & SLitrani $^{1}$ & 1.81 & SLitrani $^{1}$ \\
\hline Wavelength of emission $\max [\mathrm{nm}]$ & 440 & - & 420 & - \\
\hline Light output [photons/MeV] & $25000-32000$ & 31000 & $8000-11500$ & 8400 \\
\hline Attenuation length [cm] & 20.8 & SLitrani $^{1}$ & 30.3 & SLitrani $^{1}$ \\
\hline Radiation length & 1.14 & - & 1.38 & - \\
\hline Scintillation Yield & & SLitrani $^{1}$ & & SLitrani $^{1}$ \\
\hline Moliere radius & 2.03 & - & 2.3 & - \\
\hline Wrapping material & \multicolumn{4}{|c|}{ Teflon AF1601, $60 \mu \mathrm{m}$} \\
\hline Wrapping material of module & \multicolumn{4}{|c|}{ Mylar, $20 \mu \mathrm{m}$} \\
\hline Physical processes & \multicolumn{4}{|c|}{$\begin{array}{l}\text { Photoelectric effect, Compton and multiply scattering, gamma } \\
\text { conversion, bremsstrahlung, pair production }\end{array}$} \\
\hline Optical model & \multicolumn{4}{|c|}{$\begin{array}{l}\text { Optical absorption, Rayleigh scattering, optical scattering } \\
\text { reflection, reflection on the interaction between surfaces }\end{array}$} \\
\hline
\end{tabular}

Note: SLitrani ${ }^{1}$ means that the corresponding parameter obtained in SLitrani, and it is some function.

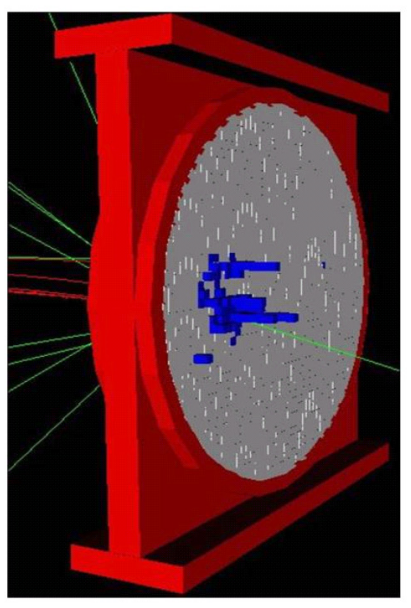

a)
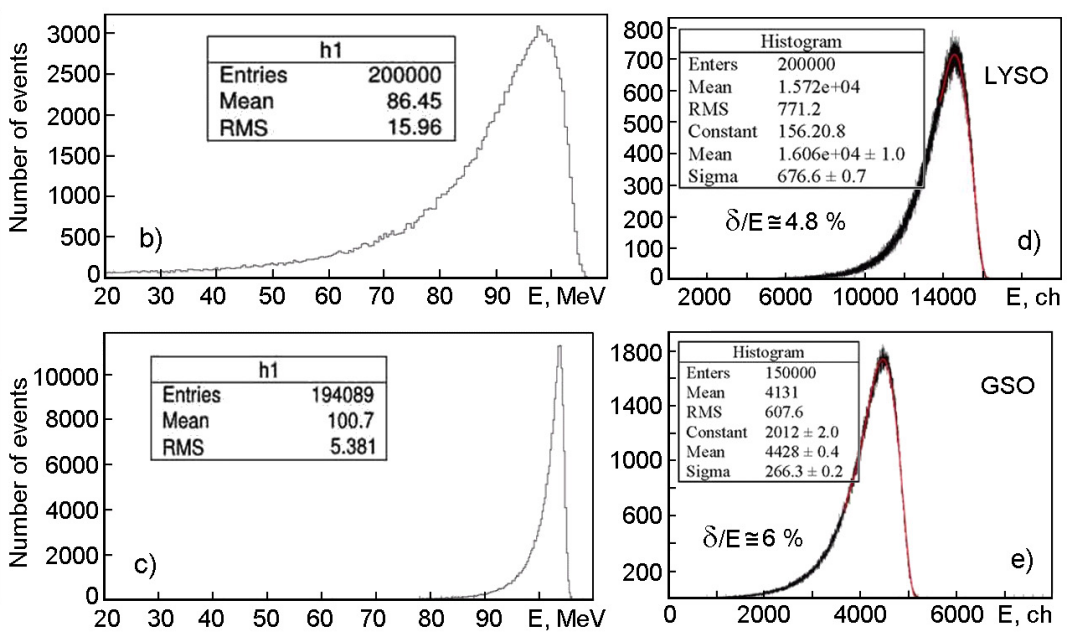

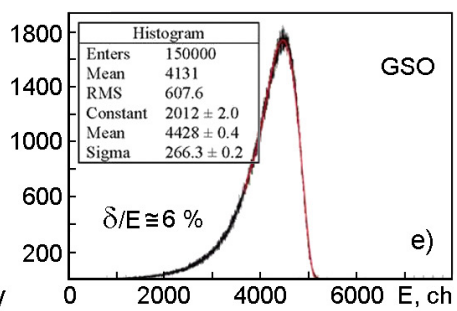

Fig. 2. Simulation of the ECAL calorimeter in $1 \mathrm{~T}$ uniform magnetic field at $105 \mathrm{MeV}$ electron beam: a) geometry of the ECAL calorimeter; b) the energy deposition of the calorimeter on GSO crystals wrapped with Teflon and grouped in modules of $2 \times 2$ crystals wrapped with Mylar; c) the energy deposition of the calorimeter on GSO crystals wrapped with Teflon; the simulated energy spectrum of the calorimeter on LYSO - d) and GSO - e) crystals.

cantly make worse the energy resolution of the calorimeter (Fig. 2b). In this case, the structure of the calorimeter becomes inhomogeneous and the losses of energy (photons) inside the calorimeter depend not only on the processes of scattering and absorption on the material of the crystal and wrapper, and it is also strongly depends on the trajectory (position) of electron beam. 


\section{Spatial reconstruction of events inside crystals of the segmented ECAL calorimeter}

To estimate the energy resolution of the ECAL calorimeter, which can be obtained on LYSO and GSO crystals the experimental measurements of the calorimeter prototypes at electron beam of accelerator in Tohoku were made. Prototypes were performed in the form of the segmented LYSO and GSO matrixes of size $7 \times 7$ crystals. Each crystal was wrapped with two layers of Teflon tape (AF 1601, one layer thickness is $60 \mu \mathrm{m}$ ) and was combined in modules wrapped with Mylar. The thickness of the Mylar is $20 \mu \mathrm{m}$. The prototypes had the following segmentation: the Mylar module from $9(3 \times 3)$ crystals in the center, the Mylar module from 6 $(2 \times 3)$ and $4(2 \times 2)$ crystals on the edges of the matrix.

Data are taken at five different electron energies: 65, 85, 105, 125 and $145 \mathrm{MeV}$ without external magnetic field. In the results of data handling was obtained the energy resolution, equal to $4.6 \%$ for calorimeter prototype on LYSO crystals and $5.7 \%$ for prototype on GSO crystals [6].

In the results of the Geant4 simulation at the $105 \mathrm{MeV}$ electron beam was obtained the energy resolution, equal to $\sim 4.8 \%$ for calorimeter prototype on LYSO crystals (Fig. 2d) and $\sim 6.0 \%$ for prototype on GSO crystals (Fig. 2e). It need be note that the value of the measured energy resolution is a bit better than simulated value. It is can be explained by the fact that:

- the Geant4 simulation of the calorimeter was made taking into account a uniform magnetic field at the $105 \mathrm{MeV}$ electron beam energy spread, and the beam test experimental result was obtained for the case when a narrow $105 \mathrm{MeV}$ beam hits at a right angle to the central crystal of prototype;

- during beam test data handling, light output losses along the crystal length were not taken into account.

When the handling of results of measurements without taking into account the losses of the light output, signals from the APD outputs, proportional to the energy deposition in the crystals, simply summed, and as result the measurement of energy will has an error. This is because the energy leakage from one crystal to another takes place at specific distances from APD and without taking into account the losses along the crystal length (photons pass only part of the crystal length), the value of these energies when calibrating on the beam will be increased compared with real values. And as a consequence, the value of total energy deposited, obtained in the calorimeter, will be large compared with realwith the same standard deviation and it will lead to a false improvement of the energy resolution of the calorimeter:

$$
\begin{gathered}
R_{\text {real }}=\frac{\sigma}{\sum E_{\text {real }}} \%>R_{\text {measur }}= \\
=\frac{\sigma}{\sum E_{\text {real }}+\sum E_{\text {error }}} \% .
\end{gathered}
$$

The value of this error $E_{\text {error }}$ strongly depends on the track inside the calorimeter and can be quite large, especially for curved tracks, when the calorimeter is located in the magnetic field.

In the COMET experiment the recorded events lies in a narrow energy region $(105.0 \pm 0.5 \mathrm{MeV})$, therefore, the ECAL calorimeter should measure the energy of an electron very accurately. To solve the problem without taking into account light output losses along the crystal length is not possible with existing methods of measurements. Therefore, to achieve needed in the experiment of the accuracy of measurement of electrons energy, it is necessary to do spatial reconstruction of events within a segmented calorimeter on long crystals.

For spatial reconstruction of events in a segmented calorimeter, the authors have developed and tested an algorithm of spatial reconstruction of events inside crystals.

The main point of the method consists in the fact that when you register the event in the calorimeter it is necessary to measure two parameters for each crystal: the amplitude distribution of the signal from the crystal and arrival time on the APD.

Knowing time of the signal propagation in the crystal and arrival time on the APD, you can determine the distance in the crystal with which the signal arrived on the APD, i.e., to determine the position of track inside the crystal.

Then, knowing the dependence of distribution of light output losses along the crystal length, it is possible to restore the true value of the energy deposited in the crystal, with a given accuracy.

Consider the method more detail. Figure 3 shows the position of the electron track passing at an angle $\alpha$ through the matrix of crystals. In this case the energy deposited $E_{e}$ will be the same for all crystals along 


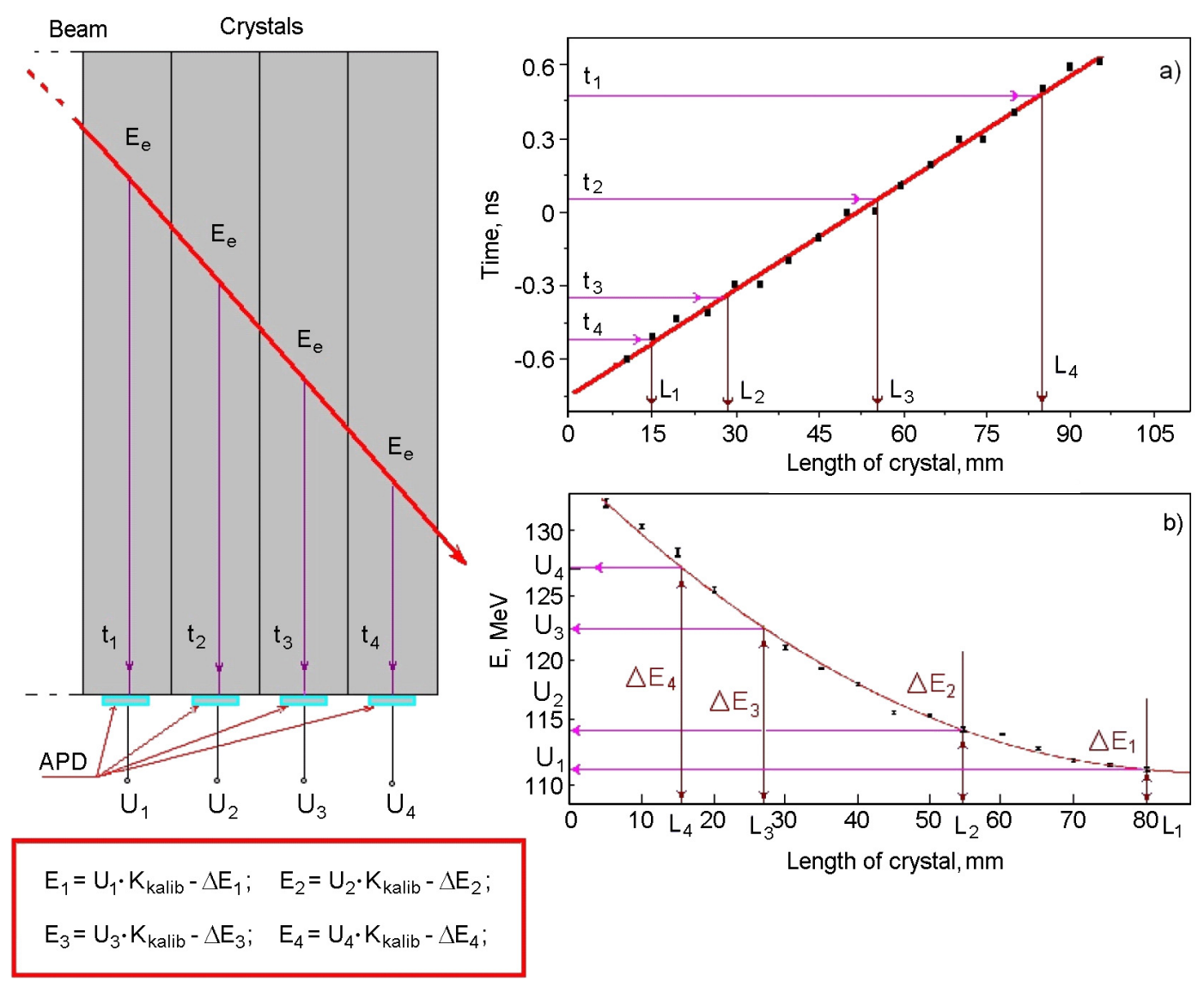

Fig. 3. The algorithm of spatial reconstruction of events inside a segmented calorimeter on long crystals; a) dependence of propagation time along the crystal length; b) dependence of the energy losses along the crystal length.

the track, but signals $U_{1} \div U_{4}$ from $t$ APD outputs (because there are the losses along the crystal length) will have different amplitude. The signal $U_{4}$ will have the maximum value of amplitude, since the distance that the photons pass from the position of track to the APD will be minimal, and the signal $U_{1}$ will have the minimum value (Fig. 3b). When preliminary energy calibration $k_{\text {kalibr }}$ of the matrix at the beam, four different values of the energy deposited $E_{i} \cdot k_{k a l i b r}$ along the track will be recorded with APD outputs for this case of the passage of electrons through the matrix, that it is not true.

The position of track (distances $L_{1} \div L_{4}$ ) in each crystal are determined by measuring the arrival time of signals $t_{1} \div t_{4}$ on APD outputs and using the obtained time dependence (Fig. 3a).

Next, using the given dependence of light output losses along the length, the errors are determined, which should be taken into account when calculating the energy deposited in crystals (Fig. 3b):

$$
\begin{aligned}
& E_{1}=U_{1} \cdot k_{k a l i b r}-\Delta E_{1} \\
& E_{2}=U_{2} \cdot k_{k a l i b r}-\Delta E_{2} \\
& E_{3}=U_{3} \cdot k_{k a l i b r}-\Delta E_{3} \\
& E_{4}=U_{4} \cdot k_{k a l i b r}-\Delta E_{4}
\end{aligned}
$$

In this case, the error of energy measurement will be minimal and will depend on the accuracy of determination of the losses and propagation time of signal along the crystal length.

Experimental verification of the methodology of spatial reconstruction of events was made on the real prototype. The prototype was performed as a $3 \times 3$ matrix of LYSO crystals $\left(20 \times 20 \times 150 \mathrm{~mm}^{3}\right)$. Crystals were wrapped with a double layer of Teflon (AF1601, thickness $60 \mu \mathrm{m}$ ) and placed in Mylar module (Fig. 4g). The thickness of the Mylar film was $20 \mu \mathrm{m}$. Readout of the signals from crystals were made using APD S866 (Hamamatsu).

All experimental measurements were carried out on cosmic muons and statistics of 

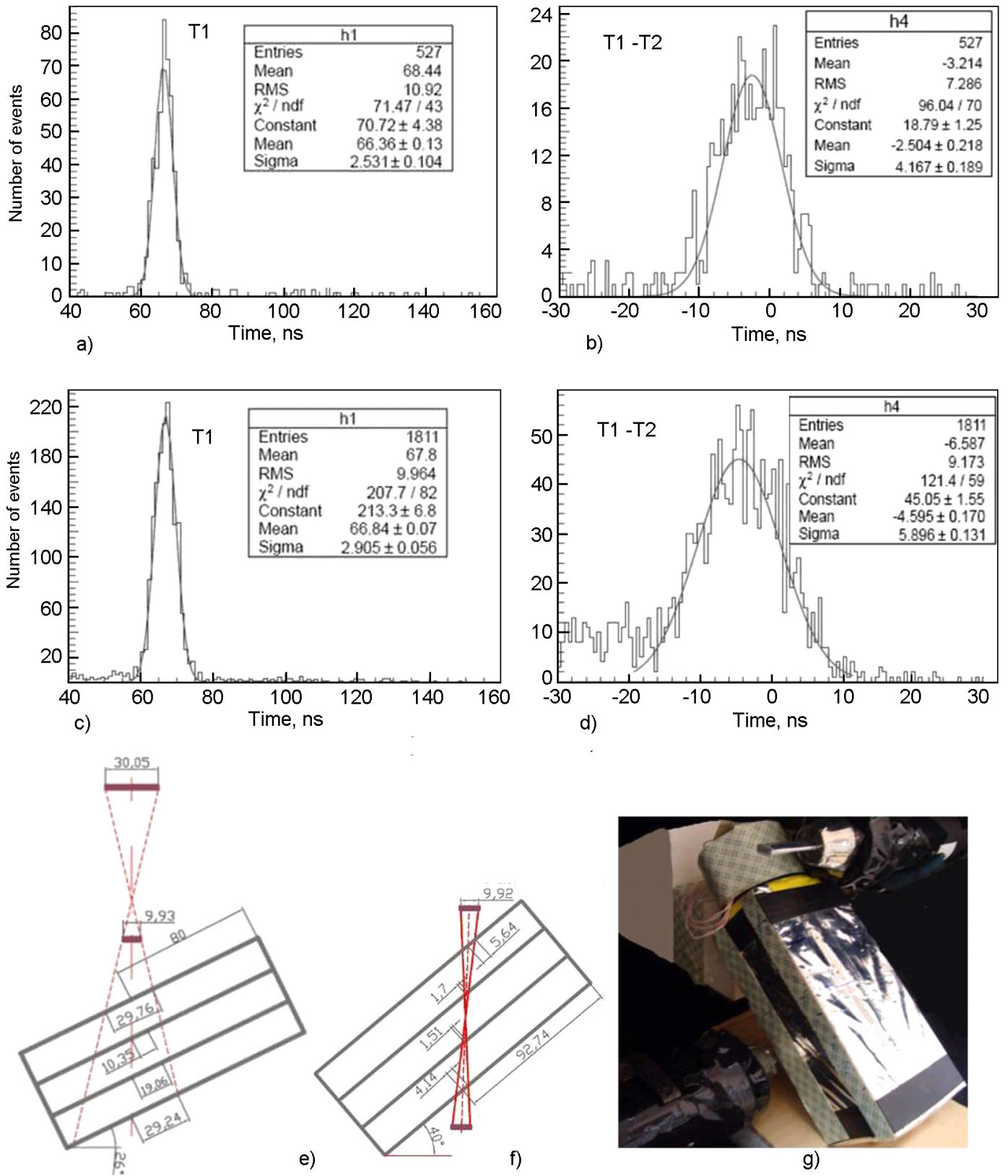

g)

Fig. 4. Experimental measurements of propagation time of the signal along the crystal length for 26 and 40 degrees angles: a) time spectrum of signal arrival from crystal \#1; b) the difference of arrival time of the signals from the crystals \#1 and \#2 on the APD inputs for the same event for 26 degrees angle; c) and d) the similar spectra as a) and b) for 40 degrees angle, respectively; e) and f) measurement scheme of signals arrival for angles 26 and 40, respectively; g) a view of the setup for measuring of 40 degrees angle.

2000 events. Measuring of light output losses along the LYSO crystals length in the prototype were making in five points at distances of $45,60,75,90$ and $105 \mathrm{~cm}$ from the APD. Figure 5a shows the dependence of light output losses along the length of the crystal \#2 in the prototype.

Measuring of the propagation time of signals along the crystal length was made for angles of rotation of the matrix of 10 , 26 and 40 degrees (Fig. 4e, f, g). Knowing the time difference of arrival of signals on APD inputs and the difference of distances from which arrived these signals was obtained the dependence of signal propagation time along the crystal length for these angles (Fig. 5c). Figure 4 shows the experimentally measured time spectra of the sig- 

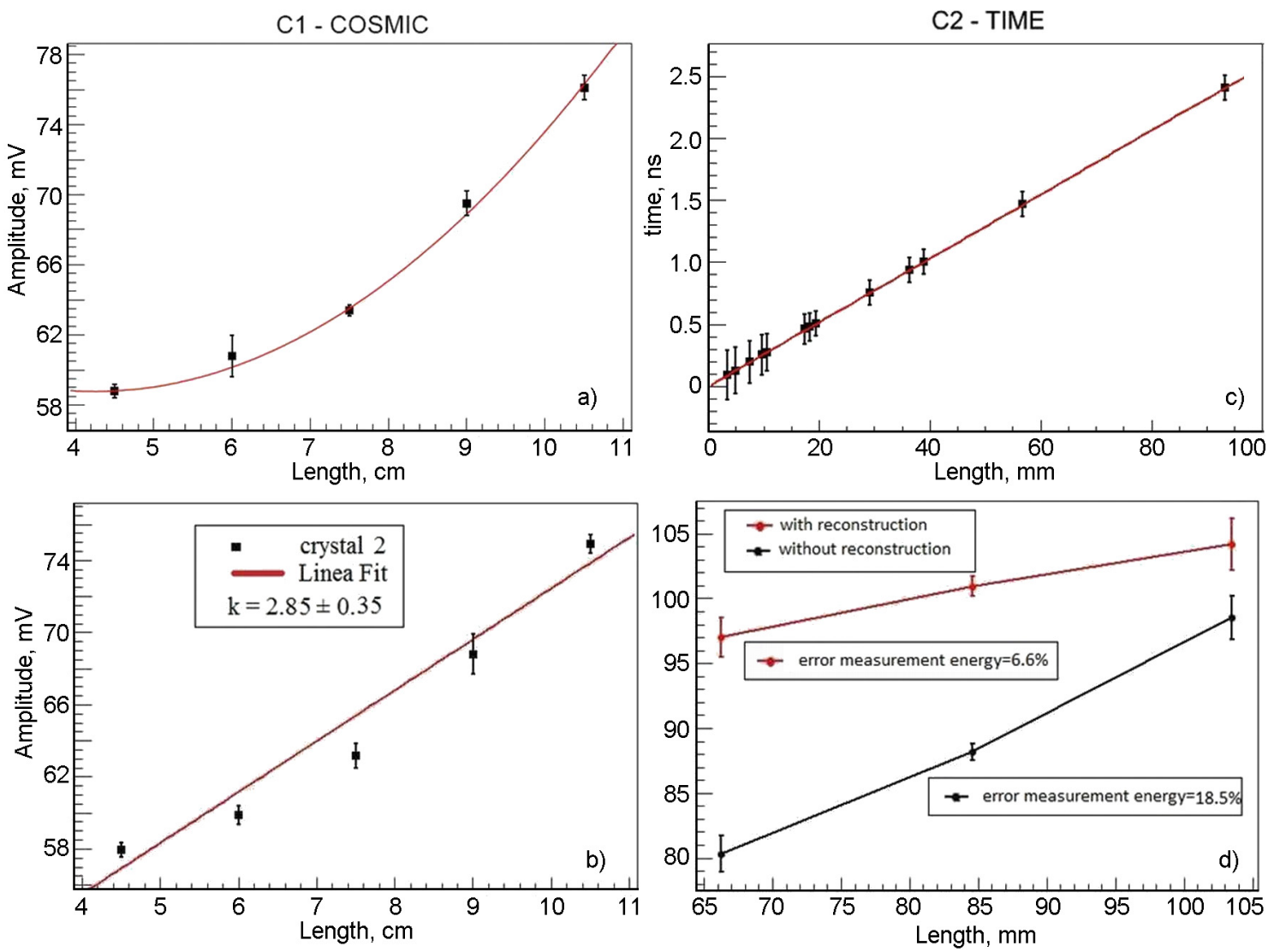

Fig. 5. The result of experimental checking of the method of spatial reconstruction of events in the calorimeter: the light output losses for crystal \#2 - a); losses coefficient for crystal \#2-b); c) the dependence of propagation time along the crystal length; d) the result of the comparison of energy measurement without taking into account (black) and with (red) of spatial reconstruction of events.

Table 5. Statistical values of arrival time and the difference of arrival time of signals on APD inputs

\begin{tabular}{|c|c|c|c||}
\hline Time, ns & $\begin{array}{c}\text { Angle of } \\
\text { 10 degree }\end{array}$ & $\begin{array}{c}\text { Angle of } \\
\text { 26 degree }\end{array}$ & $\begin{array}{c}\text { Angle of } \\
40 \text { degree }\end{array}$ \\
\hline$T_{1}$ & $67.73 \pm 0.16$ & $66.36 \pm 0.11$ & $66.84 \pm 0.06$ \\
$T_{2}$ & $68.65 \pm 0.27$ & $68.92 \pm 0.29$ & $71.64 \pm 0.08$ \\
$T_{3}$ & $69.59 \pm 0.17$ & $71.48 \pm 0.28$ & $76.37 \pm 0.07$ \\
$\Delta t_{1}-\Delta t_{2}$ & $0.923 \pm 0.32$ & $-2.50 \pm 0.19$ & $-4.59 \pm 0.17$ \\
$\Delta t_{1}-\Delta t_{3}$ & $-1.83 \pm 0.17$ & $-5.11 \pm 0.21$ & $-9.367 \pm 0.21$ \\
\hline
\end{tabular}

nals from the crystal 1 (Fig.4-a) and 2 (Fig.4-c) for angles 26 and 40 degrees and the spectra of the difference in propagation time of signals from these crystals for the same event (Fig.4b,d) at the output of the digitizer (it is direct measurement without taking into account the hardware delays of the signals in the channels read) for angles 26 and 40 , respectively.
Table 6. Mean value of the propagation time of signals depending on the distance, measured for different angles

\begin{tabular}{|c|c|c|c|}
\hline $\begin{array}{l}\text { Angle, } \\
\text { degree }\end{array}$ & $L, \mathrm{~cm}$ & $t, \mathrm{~ns}$ & $t=\frac{\Delta t_{1}-\Delta t_{i}}{L}$, \\
\hline \multirow[t]{2}{*}{10} & $L_{1}$ & 3.5 & $0.263 \pm 0.09$ \\
\hline & $L_{2}$ & 7.0 & $0.260 \pm 0.02$ \\
\hline 26 & $L_{1}$ & 9.75 & $0.257 \pm 0.02$ \\
\hline 40 & $L_{1}$ & 18.4 & $0.249 \pm 0.01$ \\
\hline \multicolumn{3}{|c|}{ Mean time } & $0.2575 \pm 0.16$ \\
\hline
\end{tabular}

The measured time parameters, based on which it was calculated average propagation time of the signal along the crystal length are given in Table 5 and 6 . Here $T_{1}, T_{2}, T_{3}$ are arrival time of the signal on APD inputs from crystals $\# 1, \# 2, \# 3$, respectively. The value $\left(\Delta t_{1}-\Delta t_{2}\right)$ and $\left(\Delta t_{1}-\Delta t_{3}\right)$ are difference of time of the arrival signals on APD inputs for the same event and $L_{1}, L_{2}$ is a 
distance in crystals from the APD to track which passes through the matrix (Fig. 4e, f).

According to the results of measurements was obtained the dependence of propagation time of the signal along the crystal length (Fig. 5c), which defines the position of a track passing of muon through the crystals of the matrix. To obtain more precise value of the photons losses along the crystal length, the losses coefficients of light output were calculated (Fig. 5b). The error which must be compensated for the results of direct measurements was determined by using these coefficients and knowing the position of the track.

In Figure 5d is shown the result of the experimental checking of method of spatial reconstruction of the track in prototype. The error of energy measurement without spatial reconstruction is $18.5 \%$ and $6.6 \%$ if we use spatial reconstruction. Thus, the improvement of the accuracy of energy deposited measurement in the segmented prototype (matrix) for cosmic muons in 2.8 times was obtained, it is very important based on the requirements of the COMET calorimeter.

Data analysis of Tohoku beam test measurements showed that the calculated values of the energy deposited in the prototype of the calorimeter for direct and track at an angle of 20 degrees differ about $10 \%$ at the energy at the $105 \mathrm{MeV}$ electron beam. This result is good agrees with the results presented in Fig. 5d, that it confirms the correctness of the method of spatial reconstruction of tracks in the segmented calorimeter.

\section{Conclusions}

As a result, of the SLitrani research and experimental measurements of optical properties of crystals was obtained real optical model of the crystal. Based on the model we simulated the optimal structure and geometry of the COMET calorimeter, the influence of the electromagnetic shower, the energy deposited and energy resolution by using the Geant4 code. Also using the Gaent4 simulation on the $105 \mathrm{MeV}$ electrons source with the use the real optical model, an energy resolution of $4.8 \%$ was obtained for calorimeter on LYSO crystals and $6.0 \%$ for calorimeter on GSO crystals.

Have been proposed and experimentally tested the algorithm spatial reconstruction of events in the calorimeter, which significantly improves results of direct measurements of the energy deposition of the segmented calorimeter, which is an important result for the COMET experiment. The algorithm of spatial reconstruction can improve the accuracy of the determining of energy deposition in the calorimeter approximately 2.8 times.

\section{References}

1. Y.Kuno, Y.Okada, Rev.Mod.Phys., 73, 151 (2001).

2. W.Bertl et al., Eur.Phys.J., C47, 337 (2006).

3. Y.G.Cui (Brookhaven) et al., KEK-2009-10, 10, 208 (2009).

4. S.Agostinelli et al., Nucl.Instr. Meth. Phys. Res., A506, 250 (2003).

5. V.Kalinnikov, E.Velicheva, Phys. Elemen. Particl. and Atom. Nucl. Lett., 11, 418 (2014).

6. Kou Oishi, $14^{\text {th }}$ COMET Intern. CM, September 22-26, 2014, Tbilisi, Georgia 140926 COMET CM14_KouOishi.

7. http://www.omegapiezo.com/crystal_scintilat tors.html; http://www.vniia.ru/rgamo/ literat/obzor/doc/obzorrus.pdf; http://www.greenstar.ru/articles/cpektromet $\mathrm{r} \mathrm{u} \mathrm{c} \mathrm{h} \mathrm{e} \mathrm{c} \mathrm{k} \mathrm{u} \mathrm{e} \mathrm{kom} \mathrm{ple} \mathrm{k} \mathrm{c} \mathrm{s} \mathrm{.} \mathrm{p} \mathrm{d} \mathrm{f} \mathrm{;}$ http://www.isma.k̄harkov.ua/eng/

8. Woo Gyo Lee et al., J.Nucl.Sci. and Technol., Supp., 5, 572 (2008).

9. V.Kalinnikov, E.Velicheva, COMET-doc-107-v2.

10. M.Tanaka et al., Nucl.Instr. Meth. Phys. Res., A, 404, 283(1998).

11. F.X.Gentit, CMS-NOTE-2001-044, (Oct. 2001); http://gentitfx.fr/SLitrani/. 